\title{
A Rare Case of Phyllodes Tumor Metastasis to the Stomach Presenting as Anemia
}

\section{Do II Choi, MD ${ }^{1}$ \\ Ho Seok Chi, MD \\ Sang Ho Lee, MD 1 \\ Youngmee Kwon, MD, PhD2,3 \\ Seog Yun Park, MD, PhD \\ Sung Hoon Sim, MD',2 \\ In Hae Park, MD, PhD', \\ Keun Seok Lee, MD, PhD ${ }^{1,2}$}

\begin{abstract}
Metastasis of a phyllodes tumor to the stomach is an extremely rare condition with important clinical implications. A 44-year-old woman was initially diagnosed with a phyllodes tumor in her right breast in 2008, and subsequently presented to an outpatient clinic with dizziness on December 16, 2013. We found that she had severe anemia (hemoglobin levels, $6.7 \mathrm{~g} / \mathrm{dL}$ ), and we quickly performed esophagogastroduodenoscopy to identify the cause. This procedure revealed large ulcerofungating masses with active bleeding in the stomach. Histopathological examination revealed that the masses were consistent with phyllodes tumor metastases. In patients with a metastatic phyllodes tumor presenting as anemia, gastric metastasis should be considered as one of the differential diagnoses because overlooking the possibility might have dire consequences if cytotoxic chemotherapy were administered.
\end{abstract}

${ }^{1}$ Department of Internal Medicine, National Cancer Center Hospital, Goyang,

${ }^{2}$ Center for Breast Cancer,

Research Institute and Hospital, National Cancer Center, Goyang,

${ }^{3}$ Department of Pathology,

Research Institute and Hospital,

National Cancer Center, Goyang, Korea

Key words

Phyllodes tumor, Stomach, Neoplasm metastasis, Anemia

Correspondence: Keun Seok Lee, MD, PhD

Center for Breast Cancer, Research Institute and Hospital, National Cancer Center, 323 Ilsan-ro, Ilsandong-gu, Goyang 10408, Korea Tel: 82-31-920-1623

Fax: 82-31-903-0455

E-mail: kslee@ncc.re.kr

Received May 3, 2016

Accepted August 11, 2016

Published Online September 1, 2016

\section{Introduction}

Phyllodes tumors, which originate from the epithelium and interstitium of the terminal duct-lobular unit in breasts, are rare neoplasms that account for $0.3 \%-1.0 \%$ of all breast tumors, $2.5 \%$ of fibroepithelial breast tumors, and $0.3 \%$ of benign breast tumors [1]. The term "phyllodes tumor" was established in 1838 by Muller, and this lesion was initially believed to be benign with no potential for distant metastasis
[2]. However, in 1931, Lee and Pack [3] reported a case of phyllodes tumor metastasis to the lungs, which revealed that these tumors could exhibit malignant behavior. Many cases of metastatic phyllodes tumors have subsequently been reported, with most metastases being discovered in the lungs [3], although a few cases have involved metastases to the kidney [2], duodenum [4], and pancreas [5].

Ordinary breast cancer with invasive ductal or lobular pathology commonly spreads to the skeleton, lungs, and / or liver. Although not frequent, some reports have described 
metastasis to the stomach from invasive ductal and / or invasive lobular breast carcinoma [6]. However, phyllodes tumor of the breast rarely metastasizes to the gastrointestinal tract, and reported cases are scarce. Therefore, we report here a rare case of phyllodes tumor metastasis to the stomach that presented as anemia and describe the clinical implications of this case.

\section{Case Report}

A 44-year-old woman visited our out-patient clinic with dizziness in December 2013. She had previously been diagnosed with a $5 \mathrm{~cm}$ phyllodes tumor in her right breast and underwent right lumpectomy with axillary lymph node dissection in August 2008 (Fig. 1), which was followed by radiotherapy. In July 2011, she underwent right total mastectomy because of local recurrence of a $2.5 \mathrm{~cm}$ phyllodes tumor in the treated breast. In August 2013, at 5 years after the initial diagnosis, chest computed tomography $(\mathrm{CT})$ revealed a $4.7 \mathrm{~cm}$ lung mass. Right lower lobectomy was performed, and the pathology results revealed a metastatic phyllodes tumor. As the risk of another recurrence was considered high, adjuvant chemotherapy was recommended, but the patient refused to undergo this treatment. Four months later, multiple liver nodules were found upon chest CT, and a liver biopsy revealed metastatic phyllodes tumor cells identical to those from the primary breast phyllodes tumor. After confirming the liver metastasis, we recommended palliative chemotherapy using an ifosfamide-containing regimen to the patient, but she refused this treatment and selected only palliative symptomatic care.

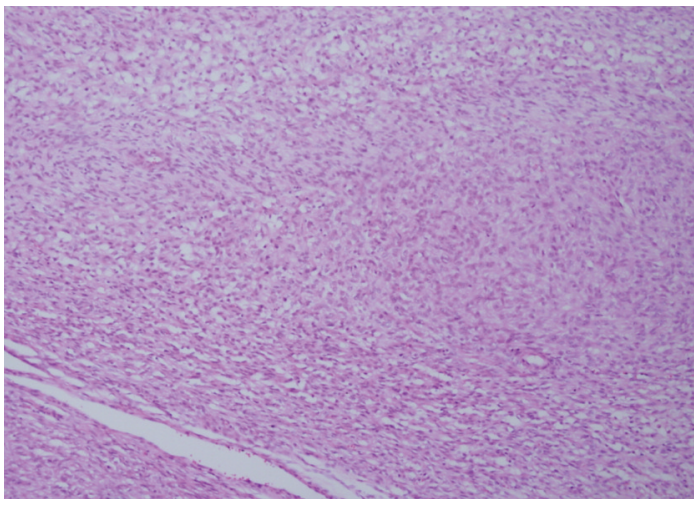

Fig. 1. Initial histopathological findings from 2008: a phyllodes tumor in the right breast (H\&E staining, $\times 100)$.

While receiving the symptomatic care, she visited our outpatient clinic because of dizziness and was admitted for further evaluation. Blood tests revealed severe anemia (hemoglobin levels of $6.7 \mathrm{~g} / \mathrm{dL}$ ), and we subsequently performed esophagogastroduodenoscopic evaluation, which identified a large gastric mass (approximately $7 \mathrm{~cm}$ in diameter) with active bleeding and several related masses (Fig. 2). Therefore, based on an initial impression of multiple metastatic gastric tumors, we performed endoscopic hemostasis with cauterization and biopsy. The biopsy confirmed that the tumors were metastases from the breast phyllodes tumor (Fig. 3). Two days later, we performed endoscopic hemostasis again for re-bleeding at the site of the gastric metastases. After the bleeding had been stopped, we considered total gastrectomy for complete bleeding control. However, we judged the patient as having a very high perio-
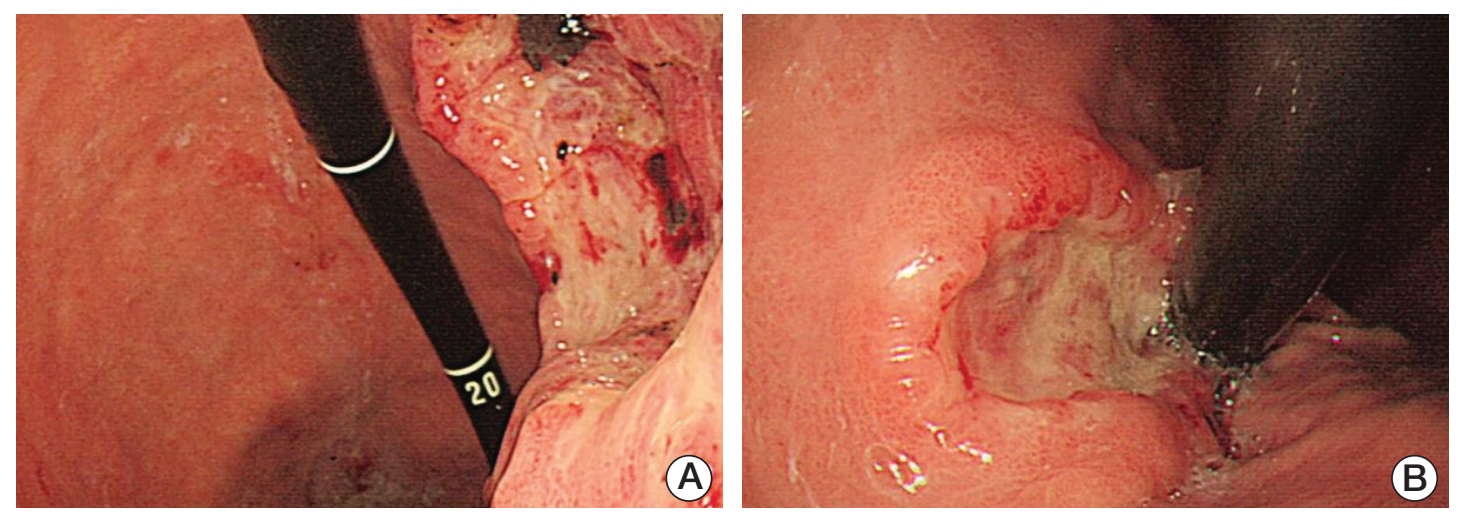

Fig. 2. Esophagogastroduodenoscopic findings: a large ulcerofungating mass in the lower body-angle (A) and the cardia side of the gastroesophageal junction on the lesser curvature (B). 


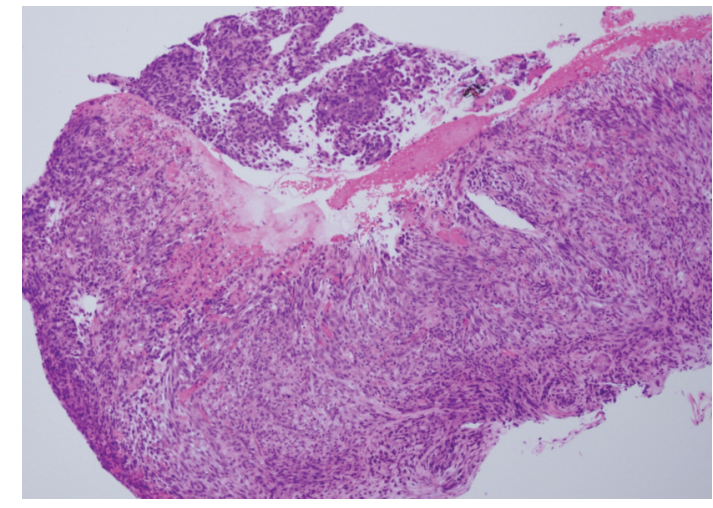

Fig. 3. Esophagogastroduodenoscopic biopsy and histopathological examination revealed sarcoma-like features that consisted of short spindle cells, which are characteristic of phyllodes tumor, and ulcerofungating gastric masses consistent with metastasis from a phyllodes tumor (H\&E staining, $\times 100$ ).

perative risk based on the rapid progression of multiple metastases involving the liver and her poor general condition. Therefore, we selected treatment using a proton pump inhibitor instead of total gastrectomy. We did not observe any evidence of re-bleeding for $>1$ week; thus, she was discharged.

Approximately 1 month after discharge, she visited our emergency room for massive melena. Esophagogastroduodenoscopic evaluation confirmed that there was active bleeding in the bed of the gastric metastasis, where we had performed the earlier hemostasis. Thus, we re-performed endoscopic hemostasis and resumed treatment with the proton pump inhibitor. The patient did not experience any other bleeding events, although she ultimately died on April 2, 2014 because of irreversible liver failure related to rapid progression of the liver metastasis.

\section{Discussion}

Phyllodes tumor, which are very rare $(0.3 \%-1.0 \%$ of all breast neoplasms), originate from the fibroepithelial connective tissue of the breast [1]. A phyllodes tumor can be classified as benign, borderline, or malignant, and is diagnosed according to the histopathological manifestations of stromal hypercellularity, cellular pleomorphism, mitotic count, the shape of the margin and the stromal pattern. The median age of onset for phyllodes tumors is 45 years [7]. Prediction of the clinical behavior of phyllodes tumors is difficult. For example, Hines et al. [8] reported that rapid tumor growth and size could not predict malignant behavior, while other studies have suggested that a tumor size of $>7 \mathrm{~cm}$ was a predisposing factor for both malignant behavior and poor prognosis [8]. In addition, it has been reported that younger patients are more likely to have a benign phyllodes tumor [9]. Furthermore, poor prognosis may be related to mixed mesenchymal components, such as osteosarcomatosis or chondrosarcomatosis [10]. Other studies have found it controversial that tumor size was related to distant metastasis [10], although positive surgical margins and a large tumor size may be significant factors for local recurrence [7].

The only curative therapy for a phyllodes tumor is complete surgical removal, including the surrounding normal tissues, and it is unclear whether chemotherapy, hormonal agents, or radiotherapy have a suitable therapeutic effect [7]. However, adjuvant radiotherapy might be useful in patients with unfavorable characteristics, such as a large tumor, high nuclear polymorphism, high mitotic index, absence of necrosis, and increased vascularity [11]. Unfortunately, there is no standard therapy for metastatic phyllodes tumors, and ifosfamide is considered the most active agent for this indication [12], although there is no evidence regarding the efficacy of hormonal therapy. Mitus et al. [13] reported an improved median survival using a combination of doxorubicin plus cisplatin, cyclophosphamide, or ifosfamide in their retrospective series of 37 patients with metastatic phyllodes tumors. Another potential treatment is sunitinib, which is an oral inhibitor of type 1 and 2 vascular endothelial growth factor receptors, platelet-derived growth factor receptors (PDGFR$\alpha, \beta)$, c-kit, FMS-like tyrosine kinase-3, and RET kinase. Sunitinib provided a major response in patients with a metastatic phyllodes tumor [14], and therefore merits further evaluation.

In the present case, a $5 \mathrm{~cm}$ malignant phyllodes tumor in the right breast was diagnosed in August 2008, and local recurrence was observed at approximately 3 years after surgical removal and radiotherapy. Metastases to the lung, liver, and stomach were subsequently observed during AugustDecember 2013. The stomach metastasis was located in the lesser curvature of the stomach, and consisted of a $7 \mathrm{~cm}$ ulcerofungating mass with multiple related nodules that presented as anemia (Fig. 2). The biopsy specimen from the stomach lesion exhibited sarcoma-like features that consisted of short spindle cells (Fig. 3), which are characteristic of a phyllodes tumor, and histopathological features that were similar to those of the primary breast specimen (Fig. 1).

The causes of anemia in patients with cancer are diverse, and include intrinsic or iatrogenic blood loss, nutritional deficiencies (primarily in iron or folic acid), hemolysis, bone marrow failure from various etiologies, infection, inflamma- 
tion, or the cancer burden itself [15]. In the present case, at the time of liver biopsy performed to evaluate the multiple liver nodules, the hemoglobin level was $11.0 \mathrm{~g} / \mathrm{dL}$ (August 2013). However, we did not perform additional evaluation of the anemia, as it was mild and asymptomatic. Nevertheless, based on the large size of the gastric mass, we assume that the stomach metastasis was present before she presented with anemia at our outpatient clinic (December 2013).

This case has two clinical implications. First, patients with a metastatic malignant phyllodes tumor and anemia should be carefully evaluated for the possible causes of anemia, which may include gastric metastasis. Second, when considering chemotherapy and/or oral sunitinib to manage a metastatic phyllodes tumor, clinicians should carefully search for any bleeding foci, as chemotherapy and / or sunitinib can increase the risk of bleeding. In the present case, the patient refused chemotherapy, although it is possible that this treatment might have had dire consequences if we administered it before identifying the gastric metastasis.

In conclusion, we report here a rare and unusual case of phyllodes tumor metastasis to the stomach, which presented as anemia. In patients with a metastatic phyllodes tumor presenting as anemia, gastric metastasis should be considered as one of the differential diagnoses. In addition, clinicians should be aware of the risk of bleeding from a hidden focus, including the stomach, when considering chemotherapy and/or targeted therapy.

\section{Conflicts of Interest}

Conflict of interest relevant to this article was not reported.

\section{Acknowledgments}

This study was supported in part by a grant from the National Cancer Center (1610610-1).

\section{References}

1. Auger M, Hanna W, Kahn HJ. Cystosarcoma phylloides of the breast and its mimics: an immunohistochemical and ultrastructural study. Arch Pathol Lab Med. 1989;113:1231-5.

2. Karczmarek-Borowska B, Bukala A, Syrek-Kaplita K, Ksiazek M, Filipowska J, Gradalska-Lampart M. A rare case of breast malignant phyllodes tumor with metastases to the kidney: case report. Medicine (Baltimore). 2015;94:e1312.

3. Lee BJ, Pack GT. Giant intracanalicular myxoma of the breast: the so-called cystosarcoma phyllodes mammae of Johannes Muller. Ann Surg. 1931;93:250-68.

4. Asoglu O, Karanlik H, Barbaros U, Yanar H, Kapran Y, Kecer $\mathrm{M}$, et al. Malignant phyllode tumor metastatic to the duodenum. World J Gastroenterol. 2006;12:1649-51.

5. Wolfson P, Rybak BJ, Kim U. Cystosarcoma phyllodes metastatic to the pancreas. Am J Gastroenterol. 1978;70:184-7.

6. Winston CB, Hadar O, Teitcher JB, Caravelli JF, Sklarin NT, Panicek DM, et al. Metastatic lobular carcinoma of the breast: patterns of spread in the chest, abdomen, and pelvis on CT. AJR Am J Roentgenol. 2000;175:795-800.

7. Parker SJ, Harries SA. Phyllodes tumours. Postgrad Med J. 2001;77:428-35.

8. Hines JR, Murad TM, Beal JM. Prognostic indicators in cys- tosarcoma phylloides. Am J Surg. 1987;153:276-80.

9. Briggs RM, Walters M, Rosenthal D. Cystosarcoma phylloides in adolescent female patients. Am J Surg. 1983;146:712-4.

10. Kapiris I, Nasiri N, A'Hern R, Healy V, Gui GP. Outcome and predictive factors of local recurrence and distant metastases following primary surgical treatment of high-grade malignant phyllodes tumours of the breast. Eur J Surg Oncol. 2001;27: 723-30.

11. Chaney AW, Pollack A, McNeese MD, Zagars GK. Adjuvant radiotherapy for phyllodes tumor of breast. Radiat Oncol Investig. 1998;6:264-7.

12. Hawkins RE, Schofield JB, Wiltshaw E, Fisher C, McKinna JA. Ifosfamide is an active drug for chemotherapy of metastatic cystosarcoma phyllodes. Cancer. 1992;69:2271-5.

13. Mitus JW, Blecharz P, Walasek T, Reinfuss M, Jakubowicz J, Kulpa J. Treatment of patients with distant metastases from phyllodes tumor of the breast. World J Surg. 2016;40:323-8.

14. Park IH, Kwon Y, Kim EA, Lee KS, Ro J. Major response to sunitinib (Sutene) in metastatic malignant phyllodes tumor of breast. Invest New Drugs. 2009;27:387-8.

15. Spivak JL. Cancer-related anemia: its causes and characteristics. Semin Oncol. 1994;21(2 Suppl 3):3-8. 